\title{
High-Temperature Dielectric Relaxation of Form III Poly(vinylidene fluoride)*
}

\author{
Shigeyoshi OSAKI** and Yoichi ISHIDA*** \\ Department of Polymer Science, Faculty of Science, Osaka University, \\ Machikaneyama, Toyonaka, Osaka 560, Japan. \\ Kaoru YAMAFUJI \\ Department of Electronics, Faculty of Engineering, Kyushu University, \\ Hakozaki, Higashi-ku, Fukuoka 812, Japan.
}

(Received October 26, 1979)

\begin{abstract}
The dielectric relaxation for form III poly(vinylidene fluoride) prepared by isothermal crystallization was observed at high temperatures. The values of $\varepsilon^{\prime}$ and $\varepsilon^{\prime \prime}$ were very large at high temperatures. $\varepsilon^{\prime}$ underwent almost a tenfold increase when the temperature was raised from $150^{\circ} \mathrm{C}$ toward the melting point of the sample $\left(198^{\circ} \mathrm{C}\right)$. The sharp drop in $\varepsilon^{\prime}$ beyond the melting point was even more striking. Also, at $100 \mathrm{~Hz}, \varepsilon^{\prime \prime}$ reached a very sharp maximum at about $180^{\circ} \mathrm{C}$. The maximum value of $\varepsilon^{\prime \prime}$ increased with the degree of crystallinity. The activation energy estimated for the high temperature relaxation was about $150 \mathrm{kcal} \mathrm{mol}^{-1}$. The dielectric relaxation, whose strength was about $2.8 \times 10^{4}$, may be due to the crystalline region. It was also made clear that the relaxation is due to the form III crystallites according to mechanical and DSC measurements. There are two possibilities for the mechanism responsible for the relaxation: (1) the orientational motion of crystallites accompanying the applied ac electric field; (2) the motion of the domain walls of the form III polar crystallites which bring about a hysteresis on the polarization-electric field diagram.

KEY WORDS Poly(vinylidene fluoride)/Dielectric Relaxation / Relaxation Strength / Polar Crystal / Orientational Motion / Domain Wall / Polarization-Electric Field Hysteresis /
\end{abstract}

It has been known that poly(vinylidene fluoride) (PVDF) has three different crystalline modifications called form I, form II, and form III. ${ }^{1-3}$ Forms I and III are called polar crystals. In either form, the molecular chains have a planar zigzag conformation and the dipole moments are perpendicular to the molecular axis and do not cancel each other out. Hence, it can be expected that form I and form III should show some characteristic electric properties. Studies of piezoelectricity, pyroelectricity, and ferroelectricity have been carried out by several

* A part of Ph.D. dissertation submitted by Shigeyoshi Osaki to Osaka University, 1976.

** Present Address: Research and Development, Kanzaki Paper Mfg. Co., Ltd., 1-11, Jokojimotomachi, Amagasaki, Hyogo 660, Japan.

*** Present Address: Ishida Denki Co., Ltd., 9-8, 1chome, Hakataekiminami, Hakata-ku, Fukuoka 812, Japan. authors. ${ }^{4-9}$ These properties are closely related to dielectric properties, and hence the study of dielectric properties is important.

Form I PVDF sample has so far been prepared by the stretching method and form III PVDF sample has been prepared by solvent casting. ${ }^{2}$ However, we found that the samples so prepared were not suitable for the study of dielectric behavior at high temperatures, because the form I sample obtained by the stretching method underwent a large thermal contraction and because the form III sample obtained by the solvent-casting method showed an anomalously large ionic conduction as the temperature was raised.

As described in a previous paper, ${ }^{10}$ we found that samples of form III PVDF, dimensionally stable at high temperatures, can be prepared by isothermal crystallization at temperatures between $165^{\circ} \mathrm{C}$ and 
$175^{\circ} \mathrm{C}$ This finding made it possible to study the dielectric relaxation of form III PVDF at temperatures near its melting point. The present paper describes the results from this study.

\section{EXPERIMENTAL}

A PVDF film, known as the KF-Polymer, was kindly supplied by Dr. H.Kakutani of Kureha Chemical Co., Ltd. The number-average degree of polymerization was stated to be approximately 1000 . Samples of form III PVDF prepared by isothermal crystallization at high temperatures are here referred to as form III (ISO). ${ }^{10}$ The samples prepared by solvent casting from an $0.1 \%$ dimethylformamide solution were designated as form III (SVT) here. The crystal form of the included crystallites was identified to be form III, according to the X-ray photographs and IR spectra. Comparative data were taken with form II (MQ) sample prepared by quenching into liquid nitrogen from the melt. Gold was evaporated onto the surfaces of the samples as the electrodes. Dielectric measurements by a mutual-inductance bridge ${ }^{11}$ and an ultra low-frequency bridge ${ }^{12}$ were carried out. Dielectric measurements under a dc$\operatorname{bias}^{13,14}$ were also made. The P-E hysteresis loop (polarization-electric field hysteresis loop) was observed by the Sawyer-Tower method, ${ }^{15}$ using an ac electric field of $0.1 \mathrm{~Hz}$. Mechanical measurements were carried out with a Rheovibron (dynamic viscoelastic meter) Model Rheo-200 manufactured by Toyo Baldwin Co., Ltd. ${ }^{16}$ The long

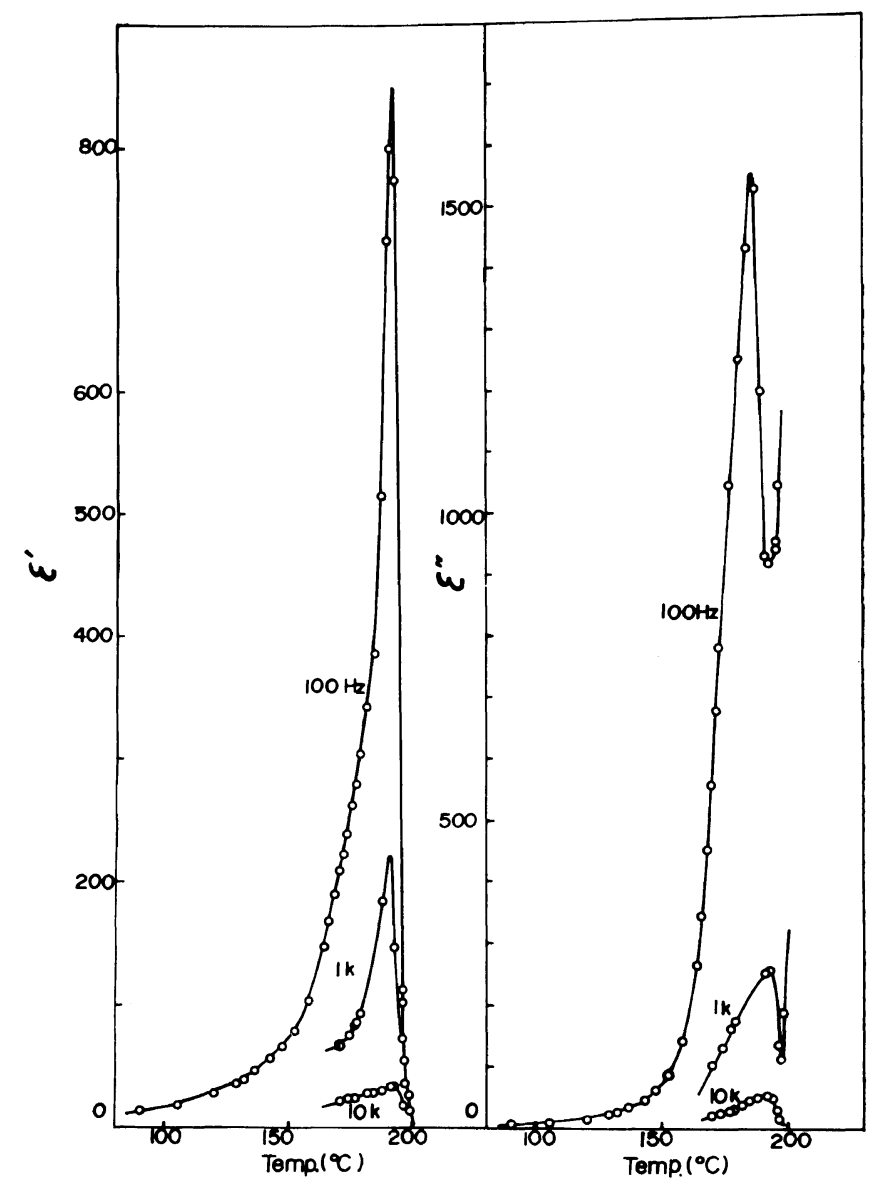

Figure 1. Temperature dependence of $\varepsilon^{\prime}$ and $\varepsilon^{\prime \prime}$ for a form III (ISO) specimen at various fixed frequencies. 
period of the samples was determined by the small angle X-ray diffraction patterns.

\section{RESULTS AND DISCUSSION}

Figure 1 shows the temperature dependence of $\varepsilon^{\prime}$ and $\varepsilon^{\prime \prime}$ for form III (ISO) at various fixed frequencies. The steep rises, both in $\varepsilon^{\prime}$ and $\varepsilon^{\prime \prime}$ with temperature, are striking. Thus, at $100 \mathrm{~Hz}, \varepsilon^{\prime}$ underwent almost a tenfold increase when the temperature was raised from $150^{\circ} \mathrm{C}$ toward the melting point of the sample $\left(198^{\circ} \mathrm{C}\right)$. The sharp drop in $\varepsilon^{\prime}$ beyond the melting point was even more striking; above $200^{\circ} \mathrm{C}$, the values of $\varepsilon^{\prime}$ almost diminished to zero. ${ }^{17,18}$ Also at $100 \mathrm{~Hz}, \varepsilon^{\prime \prime}$ reached a very sharp maximum at about $180^{\circ} \mathrm{C}$, and rose again at temperatures above $200^{\circ} \mathrm{C}$, after passing through a narrow minimum. The maxima in $\varepsilon^{\prime \prime}$ shift to higher temperatures as the frequency is increased. The sharp increase in $\varepsilon^{\prime \prime}$ above $200^{\circ} \mathrm{C}$ is attributable to ionic conduction, since this increase could be suppressed by applying a static electric field prior to dielectric measurement. Ions trapped in the crystalline portions should become mobile above the melting temperature and thus should cause an increase in $\varepsilon^{\prime \prime}$.

Figure 2 shows the frequency dependence of $\varepsilon^{\prime}$ and $\varepsilon^{\prime \prime}$ for form III (ISO) at $183^{\circ} \mathrm{C}$, and Figure 3 presents the frequency dependence of $\varepsilon^{\prime \prime}$ for the same sample

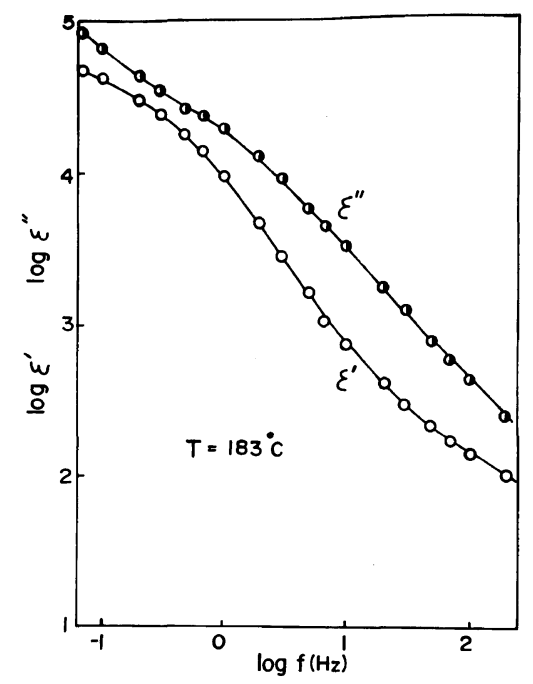

Figure 2. Frequency dependence of $\varepsilon^{\prime}$ and $\varepsilon^{\prime \prime}$ for a form III (ISO) specimen at $183^{\circ} \mathrm{C}$.

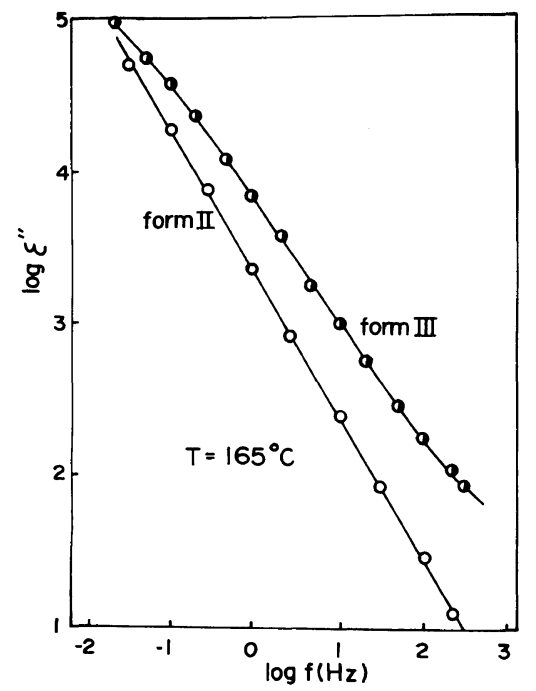

Figure 3. Frequency dependence of $\varepsilon^{\prime \prime}$ for a form III (ISO) specimen and for a form II (MQ) specimen at $165^{\circ} \mathrm{C}$.

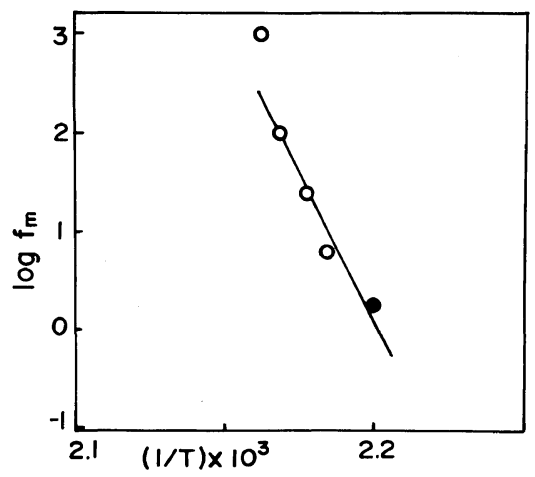

Figure 4. Arrhenius plot for the high-temperature relaxation of a form III PVDF specimen. The open circles were determined from the peaks in the $\varepsilon^{\prime \prime} v s$. temperature curves and the closed circle from the shoulder in the $\varepsilon^{\prime \prime} v s$. frequency curve at $183^{\circ} \mathrm{C}$.

and a form II (MQ) sample at $165^{\circ} \mathrm{C}$. It is seen that the $\varepsilon^{\prime \prime}$ curve for form III (ISO) is not as steep as that for form II (MQ) and has a broad shoulder. Figure 4 shows the so-called transition map for form III (ISO); the open circles have been determined from the peaks in the $\varepsilon^{\prime \prime} v s$. temperature curves and the closed circle from the shoulder in the $\varepsilon^{\prime \prime} v s$. frequency curve at $183^{\circ} \mathrm{C}$. The activation energy estimated from the indicated line is about $150 \mathrm{kcal} \mathrm{mol}^{-1}$. 
We should like to consider the following mechanisms as responsible for the marked hightemperature relaxation of form III PVDF.

1. When form III (ISO) is melted and then cooled down to room temperature, form III crystals are converted to form II crystals. In fact, the frequency dependence of $\varepsilon^{\prime}$ for a specimen obtained from the melt of a form III (ISO) specimen, displayed in Figure 5, was almost identical to that of form II (MQ). Furthermore, a form III specimen showed no absorption bands, in its IR spectrum, that could be ascribable to thermal decomposition. These facts imply that the dielectric behavior in Figure 1 should be characteristic of form III PVDF itself.

2. If this behavior were to arise from ionic impurities contained in the sample, it should be affected by the application of a static electric field. However, the values of $\varepsilon^{\prime}$ measured under a dc bias were still as large as those shown in Figure 1.

3. A semicrystalline polymer is composed of crystalline and amorphous portions. According to the theory of inhomogeneous dielectrics, interfacial polarization should cause a dielectric dispersion. If

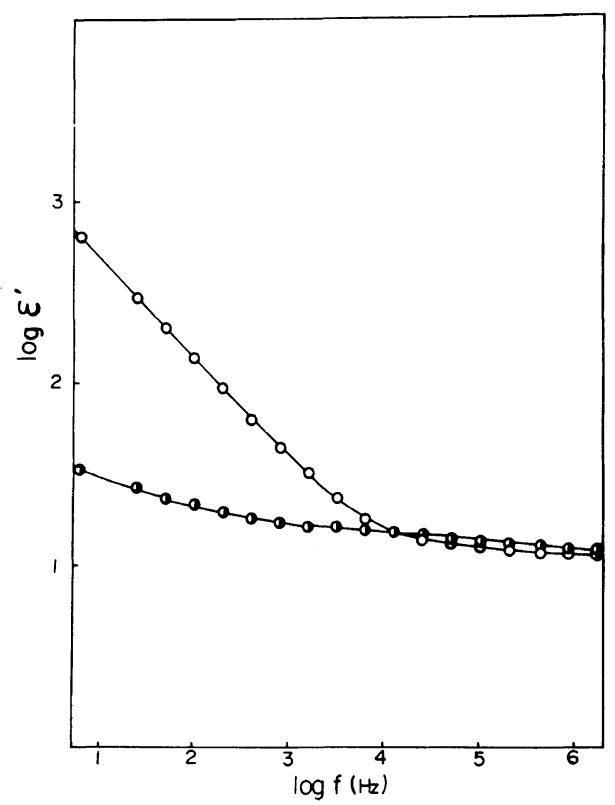

Figure 5. Frequency dependence of $\varepsilon^{\prime}$ for a form III (ISO) specimen and for a form II (MQ) specimen prepared by melting a form III (ISO) specimen; $\bigcirc$, form III (ISO); 1 , form II prepared by melting form III (ISO). $T=160^{\circ} \mathrm{C}$. we approximate the present system by a random suspension of isolated crystalline spheres of a dielectric constant $\varepsilon_{2}$ and an electric conductivity $\sigma_{2}$ immersed in an amorphous medium of a dielectric constant $\varepsilon_{1}$ and an electric conductivity $\sigma_{1}$, this effect should bring about a peak in $\varepsilon^{\prime \prime}$ at a frequency $f$ given by ${ }^{19}$

$$
f=2\left(2 \sigma_{1}+\sigma_{2}\right) /\left(2 \varepsilon_{1}+\varepsilon_{2}\right)
$$

Since, in actuality, $\sigma_{1} \gg \sigma_{2}$ and $\varepsilon_{1} \ll \varepsilon_{2}$, this may be replaced by

$$
f=4 \sigma_{1} / \varepsilon_{2}
$$

When the electric conductivity $\sigma$ of a form III (ISO) specimen at $185^{\circ} \mathrm{C}$ was measured with a dc voltage of $4 \times 10^{2} \mathrm{~V} \mathrm{~cm}^{-1}, \sigma$ reached nearly a constant value of $1.5 \times 10^{-10} \Omega^{-1} \mathrm{~cm}^{-1}$ after $15 \mathrm{~h}$, as shown in Figure 6. If this final value is used for $\sigma_{1}$ and the value of $\varepsilon^{\prime}$ at the peak for $100 \mathrm{~Hz}$ in Figure 1 is substituted for $\varepsilon_{2}$, eq 2 then gives $f \simeq 0.6 \mathrm{~Hz}$, which is a smaller value than that for the expected frequency of $100 \mathrm{~Hz}$ by a factor of $2 \times 10^{2}$. This factor, though quite crude, is large enough to eliminate the possibility the behavior in Figure 1 could be attributed to interfacial polarization.

4. It was shown in a previous paper ${ }^{10}$ that the high temperature observed near $200^{\circ} \mathrm{C}$ in the DSC thermogram of a form III (ISO) specimen is ascribable to the melting of the form III crystals, and that the enthalpy $H$ associated with such a peak may be taken as a measure of the amount of form III crystals at temperatures above $180^{\circ} \mathrm{C}$. If the relaxation obeys a Debye type of equation, it follows

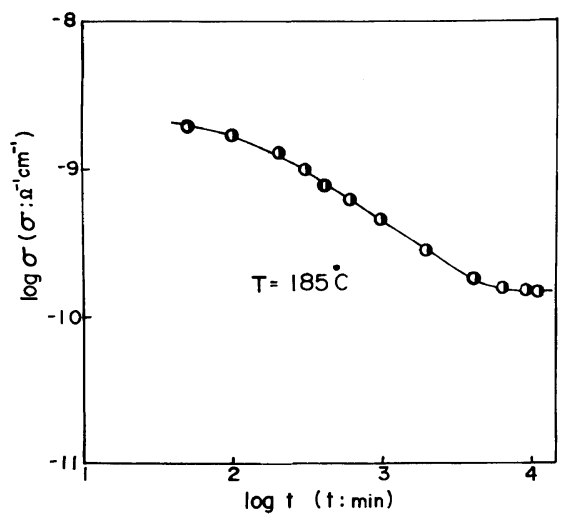

Figure 6. Time dependence of electrical conductivity at $185^{\circ} \mathrm{C}$ for a form III (ISO) specimen. 
that the relaxation strength $\Delta \varepsilon$ is proportional to $\varepsilon_{\max }^{\prime \prime}$. Although this simple assumption apparently does not hold for the high temperature relaxation under consideration, we regard $\varepsilon_{\max }^{\prime \prime}$ as a rough measure of $\Delta \varepsilon$. In Figure $7, \varepsilon_{\max }^{\prime \prime}$ at $100 \mathrm{~Hz}$ for the high-temperature relaxation curves of form III (ISO) samples crystallized at $167^{\circ} \mathrm{C}$ for different periods of time are plotted against $H$ evaluated from the DSC curves of the corresponding samples. It was observed that $\varepsilon_{\max }^{\prime \prime}$ increased monotonically with $H$, suggesting that form III crystals are mainly responsible for the marked high temperature dielectric dispersion as shown in Figure 1.

To obtain further support for this suggestion we tried to measure the mechanical loss of form III (ISO) at high temperatures, but encountered difficulties in doing so because the specimen broke with partial melting of the crystalline portions. Hence we used the form III (SVT) samples, since the

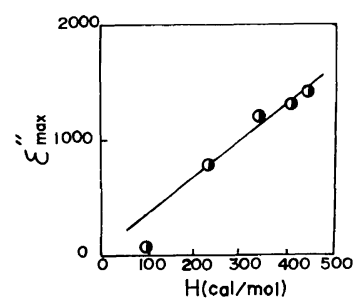

Figure 7. Relationship between $\varepsilon_{\max }^{\prime \prime}$ at $100 \mathrm{~Hz}$ and enthalpy of fusion of form III crystals crystallized at $167^{\circ} \mathrm{C}$ for different times.



Figure 8. Relationship between mechanical loss peak and long period for form III (SVT) sample. The long periods determined from small-angle X-ray photographs are given in the figure. ionic conduction affecting their dielectric behavior does not interfere with mechanical measurement. The results are illustrated in Figure 8 . The peaks in loss modulus $E^{\prime \prime}$ were seen to shift to higher temperatures as the long period of the crystal was increased. For form III (ISO), the loss peak should appear at a higher temperature than that indicated here, probably at about $180^{\circ} \mathrm{C}$, because such a sample is expected to have a long period reaching $140-150 \AA$. The expected peak temperature is comparable to the temperature for $\varepsilon_{\max }^{\prime \prime}$ in Figure 1. Such agreement, together with the fact that the feature of Figure 8 is similar to that reported for mechanical crystalline relaxation of solution-grown crystals of polyethylene, ${ }^{21}$ suggests that the hightemperature dielectric relaxation under consideration is an effect produced by the crystalline regions of form III PVDF.

However, the large strength of this relaxation can not be interpreted in terms of molecular motions associated with chain-folded lamellae. The difference between the values of $\varepsilon^{\prime}$ at $0.3 \mathrm{~Hz}$ and $200 \mathrm{~Hz}$ in Figure 2 gives a relaxation strength $\Delta \varepsilon$ of about $2.8 \times 10^{4}$ if a single relaxation mechanism is assumed. We now assume that $n$ monomer units move cooperatively in a crystallite. Then $n$ may be estimated from

$$
\Delta \varepsilon=\frac{4 \pi}{3}\left(\frac{E_{\mathrm{r}}}{E}\right) \frac{N}{n} \frac{(n \mu)^{2}}{k T}
$$

where $N$ is the number of monomer units per $\mathrm{cm}^{3}, \mu$ is the dipole moments of a monomer unit, $k$ is the Boltzman constant, $T$ is the absolute temperature, $E$ is the applied electric field, and $E_{\mathrm{r}}$ is the local electric field. If we assume that $N \simeq 1.8 \times 10^{22}, \mu \simeq 2.1 \mathrm{D}$, $T=456 \mathrm{~K}$, and $E_{\mathrm{r}} / E \simeq 1$, we find $n \simeq 6.0 \times 10^{3}$ for $\Delta \varepsilon=2.8 \times 10^{4}$. Still larger values of $n$ will be obtained if effects of crystallinity on $\Delta \varepsilon$ are taken into account. These values are too large to be acceptable physically, because the ordinary lamellar thickness is several hundred $\AA$, i.e., $n \sim 10^{2}$.

Thus, we finally come to the following two possibilities.

5. Form III crystallites are polar. Therefore, they, as a whole, may undergo orientational motions with small amplitudes when an ac electric field is applied to the specimen. This idea is not inconsistent with large values of $n$ obtained above. However, for rotational motions of crystallites as a whole, the factor $k T$ appearing in eq 3 must be replaced by 
rotational energy. This energy must be larger than $k T$, since the rotation of a crystallite may not be regarded as free thermal motion. Thus, an even larger value of $n$ is obtained when this replacement is made in eq 3 .

To check the possibility of the orientational motion of form III crystallites, we measured the dielectric polarization of a form III (ISO) specimen as a function of ac electric field. The results are shown in Figure 9, from which the saturation polarization at $0.1 \mathrm{~Hz}$ and $185^{\circ} \mathrm{C}$ is estimated to be $9.5 \times 10^{-5}$ coulomb cm $\mathrm{cm}^{-2}$. This value is larger than the one expected when all crystallites undergo complete orientation, and hence should be influenced by other factors. The saturation polarization did not decrease much when a dc electric bias of $15 \mathrm{kV} \mathrm{cm}^{-1}$ was applied for $20 \mathrm{~h}$ before measurement. The coercive electric field for the saturation curve is of the order of $100 \mathrm{kV} \mathrm{cm}^{-1}$, which is comparable to the value obtained by Tamura et al. ${ }^{9}$ for form I PVDF at room temperature.

Thus, the orientational motion of crystallites accompanying the applied ac electric field may be one of the most plausible mechanisms responsible for the marked dielectric dispersion of form III PVDF at high temperatures.

6. Besides the orientation of crystalline regions, we may consider another possibility for the region of the hysteresis loops shown in Figure 9. This is the existence of ferroelectric domains in form III crystallites. Each of such domains may have a large spontaneous polarization, and the motion of domain walls may bring about a hysteresis on the polarization-electric field diagram. If this is the case, the value of $n$ estimated above may be a little smaller

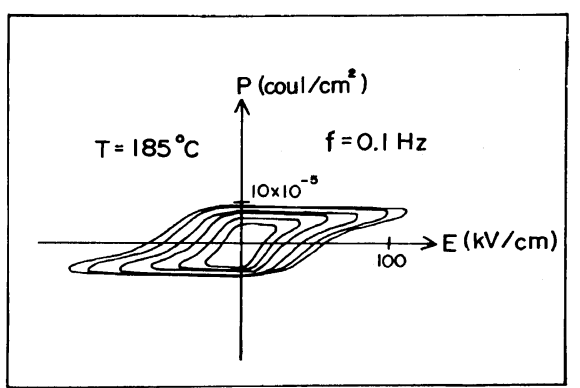

Figure 9. P-E hysteresis loop for a form III (ISO) specimen. than the actual number of monomer units contained in each form III crystallite. However, at present, it is difficult to detect experimentally the existence of ferroelectric domains in polymer crystals.

Acknowledgements. The authors are grateful to Professor Hiroshi Fujita of Osaka University for critical discussions of this work and also to Professor Tadao Kotaka of this Department for helpful discussions on constructing the manuscript.

\section{REFERENCES}

1. J. B. Lando, H. G. Olf, and A. Peterlin, J. Polym. Sci., $A-1,4,941$ (1966).

2. G. Cortili and Zerbi, Spectrochim. Acta, 32A, 2216 (1967).

3. R. Hasegawa, Y. Takahashi, Y. Chatani, and H. Tadokoro, Polym. J., 5, 600 (1972).

4. E. Fukada, Nature, 211, 1079 (1966).

5. K. Nakamura and Y. Wada, J. Polym. Sci., A-2, 9, 161 (1973).

6. M. Asahina, H. Kakutani, and K. Wada, Preprints, SPSJ, 18th Symposium on Macromolecules, Tokyo, 1969, p 449.

7. J. G. Bergman, Jr. J. H. McFee, and G. Grance, Appl. Phys. Lett., 18, 203 (1971).

8. G. Pfister, M. Abkowitz, and R. G. Crystal, J. Appl. Phys., 44, 2064 (1973)

9. Tamura, K. Ogasawara, N. Ono, and S. Hagiwara, $J$. Appl. Phys., 45, 3768 (1974).

10. S. Osaki and Y. Ishida, J. Polym. Sci., Polym. Phys. Ed., 13, 1071 (1975).

11. R. H. Cole and P. M. Gross, Rev. Sci. Instr., 20, 252 (1949).

12. S. Uemura, J. Polym. Sci., A-2, 12, 1177 (1974).

13. S. Osaki, S. Uemura, and Y. Ishida, Rep. Prog. Polym. Phys. Jpn., 8, 403 (1970).

14. S. Uemura, J. Polym. Sci., A-2, 10, 2155 (1972).

15. C. B. Sawyer and C. H. Tower, Phys. Rev., 35, 269 (1930).

16. M. Takayanagi, Mem. Fac. Eng. Kyushu Univ., 23, 41 (1963).

17. J. C. Hicks, T. E. Jones, and J. C. Logan, J. Appl. Phys., 49, 6092 (1978).

18. M. Uchidoi, T. Iwamoto, K. Iwama, and M. Tamura, Rep. Prog. Polym. Phys. Jpn., 22, 345 (1979).

19. K. W. Wagner, Arch. F. Electrotech., 2, 371, 373, 383 (1914).

20. Y. Wada, "The Physical Properties of Polymer Solids," Baifukan, Tokyo, 1971.

21. M. Takayanagi and T. Matsuo, J. Macromol. Sci. Phys., B1(3), 407 (1967). 